\title{
Los Cucapá y su medio ambiente
}

\author{
Ana A. de Williams
}

\begin{abstract}
Resumen
El objetivo de la presente investigación es señalar la manera en que los Cucapá se adaptaban al medio ambiente, enfatizando el aspecto de la obtención de alimentos y en la forma en que la vida moderna ha ido cambiando su ecosistema, y por tanto sus recursos alimenticios. El ecosistema del delta del Río Colorado, el cual en sus orígenes era caudaloso y ofrecía una gran variedad de alimentación, especialmente en su confluencia con el Río Gila, ha sufrido cambios drásticos, los cuales han afectado a la flora y fauna de esta región. Como consecuencia, sus habitantes, los indígenas Cucapá se vieron obligados a emigrar a tierras más bajas, debido a que el agua bajó tanto que ya no subían a las ricas tierras boscosas, sino solo hasta el desierto, y al igual que estas ricas regiones ellos se encuentran en grave peligro de desaparecer y además desprotegidos.
\end{abstract}

Palabras clave: Indígenas cucapá, medio ambiente, ecosistema del río Colorado, evangelización.

\begin{abstract}
This research refers to the process of adaptability of the Cucapah Indians in front of a changing environment. It also emphasizes the diverse activities they performed to obtain food and the form how modern life has affected their ecosystem; and as a result, their feeding sources. At the beginning, the ecosystem of the Colorado River delta was plentiful and offered a great variety of food products - at the point where it merged with the Gila Riverbut through the years the river suffered dramatic changes that affected the flora and fauna of this region. As a consequence, its inhabitants, the Cucapah Indians were forced to migrate to lower lands because water levels did not reach the woods anymore and the river remained only up to desert areas. The main problem is that Cucapah Indians are in danger to become extinct and, like these rich regions, they are not properly protected.
\end{abstract}

Keywords: Cucapah Indians, environment, ecosystem, Colorado River ecosystem, evangelization. 


\title{
LOS CUCAPA Y SU MEDIO AMBIENTE*
}

\author{
Por \\ Ana A. de Williams**
}

Don Juan García Aldama, el anciano de más edad entre los cucapá de río Hardy, Baja California, cuenta que existe la tradición entre su gente de recordar los tiempos cuando la sierra cucapá era una península con un gran lago de agua dulce bañando sus bajadas. ${ }^{1}$ Grandes y poderosos, el río Colorado y su hermanoel Gila, corrían desde el noroeste hacia el lago, que en aquel entonces cubría lo que hoy conocemos como los valles de Mexicali, Imperial y Yuma. La rebosadura de los ríos y del lago corría hasta el hoy golfo de California (Wide, D., 1976: 11-15).

Con los cambios de clima las lluvias disminuyeron, presentándose años de sequía hasta que el vasto lago desapareció, permaneciend o tan sólo los ríos que se unían combinando sus aguas cerca de lo que hoy es Yuma, Arizona, que serpenteaban en meandros hasta el golfo (Sykes, 1937).

Mucha gente vivió junto al lago; sin embargo, al bajar las aguas, muchos de ellos se fueron a vivir a otras partes, dejando dispersos, en las playas ahora secas, una variedad de tepalcates, utensilios de piedra, adornos de concha y seguramente otras cosas de materiales perecederos que desconocemos ${ }^{2}$ (Weide, M., 1976: 82). Algunos grupos subieron a vivir en las sierras, otros fueron hasta las costas del golfo o del Pacífico y otros a las orillas de los ríos. Los cucapá bajaron de la sierra que habitaban hasta la zona inferior del delta, que presentaba un ambiente generoso para cubrir sus necesidades ${ }^{3}$ (Rogers, 1945: 192). Las inundaciones primaverales remojaban grandes extensiones de tierra, depositando ricos sedimentos y suficiente humedad como para establecer o nutrir las plantas silvestres comestibles y el maiz, frijol y calabaza sembrados por los cucapá (Castetter y Bell, 1951: 53; Williams, 1975a: 22-34).

Los artesanos cucapá localizaban los depósitos de arcilla, misma que juntaban para ser molida y remojada, y después reposarla, para posteriormente formar y hornear ollas o trastos sencillos pero finamente elaborados. Tambiénfabricaban comales para tostar semillas, ollas de boca grande para cocinar, de boca chica para agua y unas muy grandes para almacenamiento ${ }^{4}$ (Rogers, 1945: 189).

\footnotetext{
* Este trabajo es complementodel trabajo de la autora titulado "Environment and Edible Flora of the Cocopa", publicado en: Environment Southwest, № 519. San Diego Natural History Museum. 1987. ** Investigadora especializada en estudios étnicos bajacalifornianos.

' Don Juan Garcia Aldama, anciano cucapá del río Hardy, ofreció esta información verbal a la autora.

${ }^{2}$ Hemos percibido los artefactos mencionados al caminar en varias ocasiones por las orillas de la laguna Salada y al este del cerro Centinela, Baja California.

${ }^{3}$ Información verbal proporcionada por Juan García Aldama, anciano cucapá.

${ }^{4}$ En 1976 Monserrat Sánchez, ceramista titulada, trabajó con los cucapá haciendo cerámica en la forma tradicional de ellos y nos proporcionó detalles de sus técnicas.
} 
Para la construcción de sus viviendas, los cucapá aprovechaban la madera de los bosques del delta, de los mezquites, tornillos, palofierro, álamo, sauce y de algunas plantas acuáticas como el tule y su vecina la cachanilla. Las maderas y cañas también eran utilizadas en la fabricación de sus instrumentos de trabajo tales como coas, y en armas de cacería y guerra: lanzas, arcos, flechas y dardos. Con estos objetos cazaban animales chicos como conejos y ratas, y animales grandes como el borrego cimarrón y el venado; también les permitían capturar pájaros que invernaban en las lagunas y a las orillas del río. Para la pesca usaban lanzas y redes de todos tamaños que fabricaban de fibra vegetal (Kelly, 1977).

Poco tiempo después de la desaparición de los grandes lagos, aparecieron entre los cucapá los primeros exploradores europeos. Los indígenas se alarmaron y reaccionaron hostilmente al percibir en 1540 el barco de Hernando de Alarcón. En la medida en que se fueron sintiendo seguros de que los recién llegados no presentaban amenaza alguna, los cucapá empezaron a adoptar una actitud amable; la gente del río comenzó a curiosear y a acercarse a los exploradores, cuya ropa y equipo les resultaban extraños, terminando por tratarlos como si fueran invitados de honor, ofreciéndoles maíz, panes de mezquite y cueros finamente curtidos. Los españoles por su parte, viendo que los indígenas reverenciaban al Sol, les hicieron creer que ellos eran hijos del astro rey. Los cucapá fuertemente impresionados les proporcionaron toda clase de alimentos y los defendieron de los demás indígenas. Cuando hubo necesidad de jalar los barcos españoles río arriba, su colaboración fue decidida, resolviendo el problema de los exploradores, quienes describieron a los indígenas como altos y bien formados, aspecto que aún conservan algunos de ellos en la actualidad (Williams, 1975a: 19-29).

Cuando Juan de Oñate llegó con los cucapá en 1605; sólo pudo observar a los del lado oriente del río, y sin considerar los del lado occidental, estimó que la población era aproximadamente de cinco a seis mil personas (Williams 1975a: 33-34).

Pasaron casi cien años antes de que los cucapá recibieran a sus siguientes visitantes, los misioneros jesuitas con Eusebio Kino en 1702, y 70 años después el franciscano Francisco Garcés, quien se encariñó con la gente permaneciendo con ellos un tiempo. Sin embargo, ni los cucapá ni sus vecinosestaban dispuestos a someterse a una misión; quizá era una manera de reaccionar ante los militares españoles cuyo comportamiento distaba mucho del de los religiosos a quienes acompañaban. Por tanto, la misión de Garcés terminó desastrosamente en una insurrección indígena (Williams, 1975a: 35-41).

De cualquier forma, a partir de 1785 empezóa llegar más y más gente al delta: militares, cazadores de pieles, exploradores, científicos, y para fines del siglo XIX, colonizadores (Williams, 1975a: 43-117; Herrera Carrillo, 1976: 13). A principios del siglo en curso, algunos de los cucapá ya se habían trasladado al norte, cerca de Mexicali, Baja California, y de Yuma, Arizona. Ya tenían tiempo en la colonia Lerdo y en Pozos de Arvizu (Kelly, 1977: 13), cuando los no indigenas se establecieron en los terrenos de los indígenas (Hendricks, 1976) 
tomando decisiones políticas que dividieron a los cucapá, dando por resultado que algunas familias permanecieran en Estados Unidos y otras en México (Alvarez, 1978). Con los terrenos asegurados, los colonos empezaron a manejar las aguas de los ríos usándolas para la creación de sistemas de riego, construyendo presas río arriba hasta que los cucapá ya no disfrutaban del beneficio de las inundaciones anuales que eran vitales para su agricultura, acabando así con esta actividad (Castetter y Bell, 1951: 43, 131-133, 239-240), lo cual trajo como consecuencia que el delta se secara, desapareciendo así muchos de sus alimentos silvestres. A finales del siglo pasado gran parte de los extensos mezquitales fueron cortados para dar servicio a los barcosde vapor en el río (Williams, 1975b), y para mediados de este siglo, la mayoría de los que aún quedaban se habían secado. 5

Uno de los resultados de todos estos cambios fue que los cucapá abandonaran sus costumbres alimenticias, adquiriendo una dieta similar a la de los colonos y visitantes del delta, modificaciones cuyos resultados algunos autores consideran que no fueron favorables (Calloway, 1976).

¿Qué ventajas tenían sus alimentos tradicionales, y cuáles eran sus plantas alimenticias? Aunque los cucapá contemporáneos rara vez recolectan plantas comestibles silvestres, hoy en día la gente de mayor edad entre ellos recuerda con precisión cuáles eran éstas, proporcionándonos información interesante al respecto. Veamos algunas de estas plantas para luego detenernos en una en especial.

Si hubieramos pasado por el delta del río Colorado hace unos cien años durante los meses de junio o julio, hubiésemos visto los grandes mezquitales (de Prosopis glandulosa) y a los cucapá recolectando las vainas, ya que éstas eran parte de su alimentación básica. La madera de estos árboles robustos servía para la construcción de sus viviendas, enramadas, para combustible, y para fabricar herramientas. La corteza del árbol la usaban para producir una tinta negra con la que pintaban sus cabellos y ollas. La corteza interior del árbol, remojada y machacada, se utilizaba para hacer faldas (al estilo hawaiano), y su savia seca o "chúkata" todavía se utiliza como un chicle cucapá. La flor del mezquite era una botana sabrosa, y de las vainas secas molidas en metate o en mortero se producía una harina rica para atoles y aguas frescas. También se molía y comía la semilla, la cual es rica en proteína, pero por ser muy dura se aprovechaba menos que las vainas ${ }^{6}$ (Kelly, 1977: 2-40).

Durante el verano maduraban las vainas del tornillo(Prosopis pubescens), y las del palofierro (Olneya tesota), mismas que cosechaban para ser aprovechadas en forma similar a las del mezquite. Las vainas y semillas de estos tres árboles eran valiosas porque permiten su almacenamiento sin perder sus propiedades alimenticias ${ }^{7}$ (Kelly, 1977: 32-40).

\footnotetext{
${ }^{5}$ Información verbal proporcionada por Onésimo González Saiz, líder cucapá, río Hardy 1978.

${ }^{6}$ Información verbal proporcionada por Adelaida Saiz Dominguez, Juan García Aldama, Onésimo González Saiz y Raquel Domínguez González, entre 1972-1987.

7 Ibid.
} 
Poco tiempo después de la desaparición de las inundaciones, los cucapá incluyeron al quelite (Amarantus palmeri) como parte de su alimentación, pasando a ser una verdura predilecta, que era recolectado una vez que terminaba el período de lluvias de verano, y del que también aprovechaban las semillas para la elaboración de atole y masa. Para ello molían las semillas y posteriormente le añadían agua caliente, tanto en el caso del atole como en el de la masa, y con esta última formaban tortas que tostaban en comales de barro ${ }^{8}$ (Kelly, 1977: 36-37).

Durante el mes de agosto muchos de los cucapá salían del delta con rumboal oeste, subiendo a la sierra por los arroyosy riachuelos para cosechar los frutillos de la palma (Washingtonia filifera) y de la palma azul (Brahea armata), así como los brotes tiernosde esta última ${ }^{9}$ (Castetter y Bell, 1951: 187-206). Ya en la sierra visitaban a sus amigos y parientes entre los pai pai y kumiai, además de pizcar nueces de piñón e intercambiarlas por otros productos. Posiblemente antes de bajar de nuevo a cosechar sus siembras, aprovechaban la ocasión para disfrutar allá de otros alimentos de la sierra como la nuez de jojoba y el atole de bellota (Castetter y Bell, 1951: 54-197; Alvarez y Peri, 1987: 10-15).

Durante septiembre y octubre los cucapá recolectaban las semillas de diversos pastos silvestres, entre ellos dos especies de Echinochloa y otras dos que actualmente se consideran silvestres pero que los cucapá cultivaban. De éstas últimas una era la Panicum sonorum, que se sembraba soplando la semilla hacia la tierra mojada, y la otra era la Datyloctenium aegypticum, un pasto traído del viejo mundo (Castetter y Bell, 1951: 187-190; Gifford, 1933: 267; Nabhan, 1985: 151-154).

En el invierno los cucapá se alimentaban con lo que habían almacenado durante el verano. Cortaban la calabaza y el melón en largas tiras que eran dobladas y redobladas para después colgarlas de largos palos horizontales para secarlas, o bien quebrándolas en pedazos para después almacenarlas en grandes canastas u ollas sostenidas en plataformas elevadas. De la misma manera almacenaban maiz, frijol tépari, y las vainas, semillas y raíces silvestres que habían recolectado durante el verano y el otoño. Estas plataformas no sólo servían para proteger los alimentos de los animales hambrientos, sino también de las inundaciones inesperadas, utilizando los techos como almacén. Los alimentos almacenados tenían que ser suficientes para alimentarse durante todo el invierno hasta que empezaran a madurar los de la próxima temporada de verano (Williams, 1975b: 100; Gifford, 1933: 263-267), en caso contrario, marzo y abril podían ser meses de hambre. Cuando esta situación se presentaba, los cucapá hacían viajes hasta los arroyos en dirección ascendente en busca de agaves, que sacaban con coas para después hornearlos en un hoyo (que hacían con palas de palofierro o de mezquite) revestidode piedras (Kelly, 1977: 24-41). Además, buscaban en las dunas de arena Ammobroma sonorae, planta de tallo grande subterráneo la cual tostaban en las brasas. 
Otra planta de tallo subterráneo que pudo haber sido aprovechada por los cucapá es la Pholisma sonorae, parecida a un hongo que también crece en las dunas de arena ${ }^{10}$ (Nabhan, 1985: 51-59), pero ahora es tan escasa que está en peligro de extinción; el solo hecho de encontrar una hoy en día y poder verla es digno de celebrarse.

En los meses de escasez, siempre se podia contar con las raíces del tule (Typha latifolia), las cuales si están tiernas se pueden comer crudas, y ya maduras, molidas y cocidas. También se comían la parte interior de los tallos de los tules, y el polen de la planta se usaba para endulzar otros alimentos. Además, el tule hospeda un insecto de sabor dulce que aprecian los cucapá(Kelly, 1977: 39). Por último, también disponían de otra planta de las ciénegas que los cucapá llaman "papita del agua", una variedad Sagittaria que tiene raíz comestible pero no almacenable."

Para fines de abril, el pino salado (Atriplexlentiformis) producía una semilla que los cucapá juntaban y tostaban, aunque no era de sus comidas predilectas (Kelly, 197: 39). Lo que sí gustaban y siguen disfrutando son los capullos de la biznaga (Ferocactus peninsulae), que blanquean hirviéndolos dos o tres veces para después prepararla como cualquier verdura, ya sea al vapor, en un poco de aceite, o en estofado.

Las espinas largas y fuertes de la biznaga tenían muchos usos: las calentaban y enderezaban para usarlas como agujas o leznas, o les daban forma curva para usarlas como anzuelos.

En primavera se utilizaba la Rumex violascens, que se encontraba junto al agua, de la cual se comían las hojas tiernas, y de la semilla trillada, molida y lavada varias veces (para quitar lo amargo) producían harina con la que hacían tortas y avena (Kelly, 1977: 39).

La dieta de los cucapá se componía de diversos alimentos además de los ya descritos; sin embargo, sólo mencionaremos un zacate salado que el Dr. Edward Palmer, primer científico en mencionarlo a fines del siglo pasado, le llamó Uniola palmeri, pero que es conocido por científicos contemporáneos como Distichlis palmerio Wildwheat (trigo silvestre), y comonypá o trigo gentil para los cucapá (Morán y Gould, 1981: 95-96).

Según la información proporcionada por el líder cucapá Onésimo González Saiz, existe una leyenda entre su gente que describe el descubrimiento del nypá, que se remonta a los días en que el gran lago estaba secándose: Kinakul, una persona-montaña (según la leyenda, las montañas eran gente en aquel entonces), envió a Jiesh el camaleón hasta la laguna Salada, quien a las orillas del río

\footnotetext{
${ }^{10}$ No está reportada por los cucapá, pero ha sidoposible observarla en lazona frecuentada porellos, al norte del golfo, cerca del nypá.

11 Identificado por Gary Nabhan en 1986 y confirmado por Adelaida Saiz Dominguez, Juan Garcia Aldama, Onésimo González Saiz y Raquel Dominguez González.
} 
descubrió la planta nypá ya lista para ser cosechada. Jiesh juntó unas espigas y las pegó en su cabeza y ésta fue la señal de que las aguas se habían retirado y que la nypá era buena para comer. El camaleón, que come hormigas rojas, todavía hoy en día lleva las señales de las espigas de nypá en su cabeza' ${ }^{12}$ (Sherbrooke, 1981).

Generalmente era en mayo cuando los cucapá hacían sus viajes a la boca del río para la cosecha del nypá. Viajaban varios días en pangas grandes hechas de cañas de tule amarradasen manojos con las hojas del mismo tule. Algunas de las pangas eran suficientemente grandes como para acomodar a toda una familia y transportarla durante varios días. En un extremo de la panga hacían con arcilla un piso donde ponían una pequeña fogata para poder cocinar, y la panga era manejada con un palo largo (Gifford, 1933: 272; Kelly, 1977: 34-36; Williams, 1975b: 115).

$\mathrm{El}$ ingeniero Jacobo Blanco, quien conoció la zona cuando la línea internacional fue trazada en 1863, observó los campos de nypá describiéndolos como tierras bañadas por las mareas, cubiertas con un zacate salado formando grandes praderas. Tal y como lo relató el Dr. Palmer en 1889, Jacobo Blanco describió a los cucapá que cosechaban nypá (Alvarez, 1978; Vassey, 1889), quienes tenían que hundirse casi hasta las rodillas para cosechar este zacate. Colocaban las espigas en canastas, secándolas para separar las cáscaras de las semillas. Otras personas han comentado sobre los tremendos callos que tenían los cucapá en las plantas de los pies, ${ }^{13}$ que les permitian pisar el nypá, ya que es filoso y cortante. Palmer también mencionó que la acción de las mareas facilitaba la recolección de esta planta, ya que el mar llevaba los granos flotantes a tierra firme, depositándolos en hileras onduladas, situación que los cucapá aprovechaban.

La Distichlis palmeriy la demás vegetación del delta sufrió cambios drásticos durante la primera mitad de este siglo. Las presas y los sistemas de irrigación ubicados río arriba, poco a poco permitieron controlar y aprovecharel agua del río Colorado (Sykes, 1937: 47-107). Esta actividad, junto con períodos prolongados de sequía, redujo la abundante flora del delta convirtiendo esta región en una zona de aridez desolada. En los años cincuenta los cucapá vieron desaparecer para siempre los últimos grandes mezquitales del delta. El grande y fuerte Colorado se había reducido a casi nada.

Por ser tan singular la flora y la fauna del delta del río Colorado, y por constituir una zona de reproducción importante de las especies comerciales explotadas en el golfo de California, fue declarado "zona de refugio ecológico" por decreto que apareció en el Diario Oficial del 22 de febrero de 1955.

\footnotetext{
12 Información verbal proporcionada por Adelaida Saiz Domínguez, Juan Garcia Aldama, Onésimo González Saiz y Raquel Dominguez González.

${ }^{13}$ Información verbal proporcionada a la autora por lla Gracia Bárcenas de Alvarez en la ciudad de Mexicali, en el año de 1956.
} 
Sin embargo, la zona sigue desprotegida. ${ }^{14}$ Tomando esto en cuenta y pidiendo que se reconociera el legado prehistórico e histórico del delta, en enero de 1987 un grupo de científicos mexicanos se dirigieron al director de la Comisión de Ecología de la Secretaría de Desarrollo Urbano y Ecología, solicitando el restablecimiento del santuario para la flora y la fauna del delta. Sin embargo, nada se ha hecho al respecto. ${ }^{15}$

La naturaleza misma se ha esforzado en sanar el delta. Hace aproximadamente unos diez años cayeron nieves y lluvia fuera de lo normal en las sierras que alimentan al Colorado y al Gila, y a pesar de todas las presas y sistemas de irrigación, llegaron una vez más las inundaciones al delta, con suficiente volumen como para volver a formar un lago en la depresión de la laguna Salada. La flora del delta empezó a restablecerse (siempre se inicia con pastos), y ciertos científicos se interesaron en los zacates salados del delta. ${ }^{16}$ Pero con todo y esto, todavía hace aproximadamente seis años, un respetado científico declaró en una conferencia en Arizona que el valioso Distichlis palmeri se había extinguido. $\mathrm{Y}$ en verdad, hasta para los cucapá el nypá era ya un alimento de recuerdos, no cosechado por ellos desde hace dos o tres generaciones.

Sin embargo, dos científicos dedicados, el ecólogo marino Dr. Nicholas Yensen y la nutrióloga Susana Bojórquez de Yensen, encontraron Distichlis palmeri y tienen ahora aproximadamente 16 años investigándola, señalando que existen más de 50 variedades de esta planta. Sus semillas miden de 6 a 12 $\mathrm{mm}$. El Dr. Yensen ha experimentado con algunas de las variedades - las de mayor tamaño, de múltiple espiga y con tallo de cierta fuerza y tamaño- $y$ ha podido domesticar con éxito esta planta holófila, para cuyo cultivo el agua salada es favorable, incluso la de mar $^{17}$ (Yensen, 1980: 1-31).

Existen terrenos sembrados en varias partes del mundo que son altamente productivos, pero el problema que presentan reside en su lejanía de las personas más necesitadas, ya que una cuarta parte del costo del aprovisionamiento de grano corresponde a los fletes para enviarlo a las áreas de consumo (The New Yorker, del 11 de marzo de 1985). De ahíel gran valor que implica poder utilizar terrenos salados cerca de la gente que padece hambre (Gabel, 1979).

Bien puede ser que los científicos arriba mencionados hayan salvado de la extinción a este alimento silvestre tan valioso para los antiguos cucapá. Las inundaciones del Colorado en los últimos años han desaparecido, y si los

\footnotetext{
14 En junio de 1987, más abajo de punta Invencible, pudimos presenciar a algunos pescadores aprovechándose del "burro con chinchorros" atravesados por el río. Además citamos dos cartas del Dr. Alam Weisman, de Prescott College, Arizona, describiendo las actividades pesqueras ilegales en el delta durante 1985, a pesar de las advertencias de las autoridades.

is Silvia Manzanilla Naín, carta dirigida al Lic. Alor Calderón, Comisión Nacional de Fcologia, SEDUE. 28 de junio de 1987. Véase el Proyecto Preliminar de Santuario del Delta del Rio Colorado, Alto Golfo de California, México. 1987. Silvia Manzanilla Naín.

16 Ellos son Nicholas y Susana Yensen, Amadeo Rea y Richard Felger.

17 Información verbal proporcionada por Nicholas y Susana Yensen durante varias reuniones llevadas a cabo entre 1980 a la fecha.
} 
ingenieros logran su propósito, jamás se volverá a inundar el delta del río. ${ }^{18}$ ¿Sobrevivirán entonces las plantas silvestres originales de Distichlis palmeri? No sabemos. Pero a los cucapá, entre otros, se les ha ofrecidola oportunidad de participar en el cultivo de su alimento silvestre tradicional. ${ }^{19}$

Susana Bojórquez de Yensen, al analizar la planta en busca de sus cualidades nutritivas, la encontró baja en sales, a pesar de que las hojas y los tallos de esta planta absorben y estrujan sal, reduciendo así la salinidad de la tierra. El grano es de alto contenido de fibra y bajo en fitatos antinutritivos (sales de ácido fitatético $\left.\mathrm{C}_{6} \mathrm{H}_{8}\left(\mathrm{OPO}_{3} \mathrm{H}_{2}\right)_{6}\right)$, con un contenido proteínico casi igual que el trigo común (triticum sp.). La Dra. Yensen, mediante pruebas realizadas, advirtió que el trigo gentil está libre de gluten, sustancia que parece causar alergias en ciertas personas; sin embargo, no necesitade él, pues la masa que se produce con el trigo gentil es perfectamente elástica y cohesiva para fines de panadería y repostería, además de tener buen sabor. Los Drs. Yensen afirman que según datos de la FAO (Organización para la Alimentación y la Agricultura), existen 1,547 millones de hectáreas de la superficie terrestre que no son susceptibles de cultivarse debido a su alto contenido salino. En este continente en particular, se pierden miles de hectáreas cada año por exceso de salinidad. En virtud de que las halófilas no solamente se dan en condiciones saladas, sino que remueven las sales de la tierra, los Yensen opinan que estas plantas peculiares son esenciales para el futuro de la agricultura. El Dr. Yensen ha participado en experimentos con halófilas de todo el mundo y opina que las variedades de nypá usadas por los cucapá, tienen características combinadas que pueden servir como alimento básico en el futuro próximo (Yensen, 1980). Convencidos de la importancia de la Distichlis palmeri para los paises en desarrollo, los Drs. Yensen se han organizado bajo el nombre de "Nypá" para proporcionar información y tecnología a quienes lo soliciten. Por lo pronto, tienen convenios con las universidades de Arizona y Sonora para siembras e investigación; en donde han sembrado diversas variedades de esta planta para la cosecha del grano, así como para el uso del pasto como forraje para alimentar ganado, y otro tipode pasto más bajo que puede servir para los centros recreativos campestres.

Empezamos este comentario sobre la Distichlis palmeri con una leyenda cucapá y finalizamos con otra, apuntada por el lingüista James Mack Crawford en un cuento que puede ser profético:

\footnotetext{
${ }^{18} \mathrm{Hemos}$ podido observar la disminución de las aguas del río Colorado, de la laguna Salada y del río Hardy, especialmente durante los dos últimos años; y se tiene conocimiento, a través de los periódicos de Mexicali y de Estados Unidos, de que existe el proyecto para llevar aguadel río Colorado a Tijuana, Ensenada y a ciudades del sur de California. F ara conocer el origen de esas aguas véase: Houhton, Samuel G. A Trace of Desert Waters, the Great Basin Story, y Evolution of the Colorado River in Arizona, An Hypothesis Developed at the Symposium on Cenozoic Geology of the Colorado River Plateu in Arizona, agosto 1964, editado por Edwin D. Mckee, Richard F. Wilson, William J. Bredd y Caron S. Breed. Un ejemplo vívido de los efectos de las manipulaciones de las aguas de los ríos se encuentra en el libro Once a River, de Amadeo Rea.

${ }^{19}$ Los Drs. Yensen han llevado semilla y plantas a ciertas familias cucapá del río Hardy, han llevadoa uno de sus líderes a conocer un campo experimental de Distichlis palmeri que estaba en el ejido Michoacán de Ocampo,y han ofrecido trigo gentila los cucapá de la reservacióncercana a Somerton, Arizona, en varias visitas efectuadas entre 1978 y 1987.
} 
En este mito Kma. sut. malay, uno de los amigos caníbales, ha tomado una decisión. Quiere cambiar su manera de ser. Dice que ha hecho cosas malas, pero que ya va a dejar de hacer todo esto, y que es su intención pensar en otras cosas. Quiere pensar y hacer cosas buenas. Después avisa a la gente que va a sembrar trigo, y que la gente lo va a disfrutar y comer. Definitivamente va a dejar de hacer cosas malas para en su lugar hacer y decir puras cosas buenas. Se reúne la gente y juntos siembran trigo en Makanymam, cerca del golfo, junto al río Colorado. Según él, las generacıones del futuro comerán este trigo. Cuando el trigo madura el caníbal cucapá reformado, Kma. sut. malay, reúne a la gente para la cosecha, para moler el trigo y para hacer atole y tortillas. La leyenda termina con una invitación de $\mathrm{Kma}$. sut. malay para todos: Que vengan a comer (Crawford, 1983: 67-69). 


\section{BIBLIOGRAFIA}

ALVAREZ, Ila. 1978. “Document № 35: A Map Maker’s Story of His Journey from San Diego to the Sea of Cortes”. En: Brand Book № 5. San Diego Corral of the Westerners. San Diego.

ALVAREZ, Susan H. y David W. Peri. 1987. "Acorns the Staff of Life". En: News from Native California. Vol. 1, № 4, Heydey Books, Berkeley.

CALLOWAY, D.H. 1976. "Food Patterns and Food Assistance Programs in the Cocopah Indian Community". En: Ecology of Food and Nutrition. Vol. 5. Dept. of Nutritional Science. University of Calif., Berkeley.

CASTETTER, Edward F. y W.H. Beel. 1951. Yuman Indian Agriculture: Primitive Subsistence on the Lower Colorado and Gila Rivers. University of New Mexico Press, Albuquerque.

CRAWFORD, James M. 1966. The Cocopa Language. Tesis doctoral en antropología, University of California, Berkeley.

1978. "More on Cocopa Baby Talk". International Journal of American Linguistics. 44 (1).

1983. Cocopa Texts, University of California Press, Berkeley.

GABEL, Medard. 1979. HO PING: Food for Everyone, Strategies to Eliminate Hunger on Spaceship Earth. World Game Laboratory. Anchor Books/Doubleday Garden City, N.Y.

GIFFORD, Edward W. 1933. "The Cocopa". University of California Publications in American Archaeology and Ethnology. 31 (5) Berkeley.

HENDRICKS, William O. 1976. "The Lost Cucapá Reservation". Pacific Coast Archaeological Society. Quarterly Vol. 12 (1). Costa Mesa.

HERRERA Carrillo, Pablo. 1976. Colonización del valle de Mexicali. UABC. Mexicali, B.C.

HOUGHTON, Samuel G. 1976. A Trace of Desert Waters. The Great Basin Story. The Arthur H. Clark Company, Glendale.

KELLY, William H. 1977. "Cocopa Ethnography”. Antropological Papers of the University of Arizona. 29. Tucson, Arizona.

MORAN, Reid \& Frank W. Gould. 1981. "The Grasses of Baja California, México". San Diego Society of Natural History Memoir. 12. San Diego.

NABHAN, Gary. 1985. Gathering the Desert. The University of Arizona Press. Tucson, Arizona.

1987. Native Foods of Desert People Found to Control Diabetes. The Seedhead News, № 19. Native Seeds/SEARCH, Tucson, Arizona. (ed). 1983. "The Desert Tepary as a Food Resource", a journal symposium. Desert Planis. Vol. 5 (1), University of Arizona for Boyce Thompson Arboretum, Superior AZ.

REA, Amadeo M. 1983. Once a River Bird Life and Habitat Changes on the Middle Gila. University of Arizona Press.

ROGERS, Malcolm. 1945. "An Outline of Yuman Prehistory”. Southwestern Journal of Anthropology. University of New Mexico Press. Albuquerque.

SHERBROOKE, Wade C. 1981. Horned Lizards Unique Reptiles of Western North America. Southwest Parks and Monuments Association. Earl Jackson Ed. Globe, AZ. 
SYKES, Godfrey. 1937. "The Colorado Delta". American Geographical Society Special Pub. 19. W.L.G. Joerg Ed. Carnegie Institution of Washington and the American Geographical Society of New York.

VASSEY, George. 1889. "New or Little Known Plants: Uniola Palmeri" Garden and Forest.

WILliamS, Anita Alvarez de. 1974. The Cocopah People. Phoenix: Indian Tribal Series.

1975a. Travelersamong the Cucapá. Dawson's Book Shop. Los Angeles. 1975b. Primeros pobladores de la Baja California: Introducción a la antropología de la península. Talleres gráficos del Estado. Mexicali, B.C. 1983. "Cocopa". Handbook of North American Indians. Vol. 10. William C. Sturtevant ed. Smithsonian Institution. Washington, D.C. 1987. "Environment and Edible Flora of the Cocopa". Environment Southwest. № 519. San Diego Natural History Museum, San Diego, California.

WIDE, David L. 1976. "Regional Environmental History of the Yuha Desert". En: Background to Prehistory of the Yuha Desert Region. Ballena Press. Anthropological Papers № 5. Ramona, Ca.

WEIDE, Margaret L. 1976. “A Cultural Sequence for the Yuha Desert" En Background to Prehistory of the Yuha Desert Region. Ballena Press Anthropological Papers № 5. Ramona, Ca.

YENSEN, Nicholas P. 1980. Summary of Halophyte an Hidrocarbophytes Search in Mexico, Venezuela, Brazil, Argentina, Peru. Equador \& Colombia.

YENSEN, Susana B. 1984. The Nutritional Value of a Halophytic Plant: Distichlis palmeri-vasey. Tesis de maestría. University of Arizona en Tucson. 